\title{
La gestión integrada de los recursos hídricos como estrategia de adaptación al cambio climático
}

\author{
Martha L. García-González*, Yesid Carvajal-Escobar*^, Henry Jiménez-Escobar* \\ * Escuela de Ingeniería de Recursos Naturales y del Medio Ambiente, \\ Universidad del Valle, Cali, Colombia \\ §e-mail: yecarvaj@univalle.edu.co
}

(Recibido: Abril 7 de 2006 - Aceptado: Marzo 27 de 2007)

\begin{abstract}
Resumen
La variabilidad climática (VC) y el cambio climático (CC) han sido asociados a cambios globales o a cambios en el régimen climático y han mostrado notables efectos en el ciclo hidrológico. La escasez de agua, los cambios en la intensidad de la precipitación y la variabilidad de eventos extremos están cobrando una importancia significativa. Como consecuencia del aumento de la temperatura del aire y cambios en la precipitación, el CC está incrementando la competencia por el agua dulce y está afectando los procesos y regímenes hidrológicos, generando en algunos casos un mayor flujo de contaminantes y sedimentos en lagos y ríos, degradación de la calidad del agua, alteración de la velocidad de los procesos biogeoquímicos y reducción de la concentración de oxígeno disuelto. La necesidad de adaptarse al CC aparece cuando se están produciendo grandes cambios económicos y sociales, que influyen en la utilización de los recursos hídricos (RH). El CC está aumentado la vulnerabilidad en sistemas hídricos mal administrados, haciendo urgente la necesidad de construir una nueva visión de gestión de los RH. En este articulo, se presenta el concepto de gestión integrada de los recursos hídricos (GIRH) que está ganando aceptación como una estrategia de adaptación al CC.
\end{abstract}

Palabras clave: Cambio climático, Variabilidad climática, Manejo recursos hídricos, Adaptación, Vulnerabilidad, Riesgos naturales, Prevención de desastres.

\section{Integrated water resource management as a strategy for adaptation to climate change}

\begin{abstract}
Climate variability (CV) and climate change (CC) have been associated to global changes or to changes in the climate regime and have shown remarkable effects on the hydrological cycle. Water shortage, changes in precipitation intensity, and variability of extreme events are gaining more importance. Because of the increase in air temperature and the changes in precipitation, $\mathrm{CC}$ is increasing the competition for fresh water and is impacting hydrological processes and regimes, causing in some cases a larger flow of pollutants and sediments in lakes and rivers, water quality degradation, alteration of the rate of bio-geochemical processes, and reduction of dissolved oxygen concentration. The need for adapting to $\mathrm{CC}$ is urgent when large economic and social changes are occurring that affect usage of water resources (WR). CC is increasing the vulnerability of badlymanaged water systems, creating an urgent need to build a new framework for the management of WR. In this paper, an integrated water resource management concept (IWRM) is presented, which is gaining acceptance as a CC adaptation strategy.
\end{abstract}

Keywords: Climate change, Climate variability, Water resource management, Adaptation, Vulnerability, Nature risks, Disaster prevention. 


\section{Introducción}

Tanto la variabilidad climática (VC), producto de las fluctuaciones del clima durante períodos de tiempo cortos (años, meses), como el cambio climático (CC), referido a cambios en períodos de tiempo largos debidos a variabilidad natural o a actividad antrópica están cobrando una importancia significativa. De acuerdo con las investigaciones, estos cambios obedecen mayormente a que el hombre está incrementando de manera exponencial los niveles de emisiones de gases de efecto invernadero (GEI), principalmente dióxido de carbono (IPCC, 2001b). La emisión de estos GEI y otros gases, como el metano y el óxido nitroso, están causando un impacto sobre la atmósfera, contribuyendo a la alteración de los patrones globales del clima. Los impactos específicos del CC son difíciles de identificar y el debate continúa acerca de cuáles patrones de cambio obedecen a las emisiones de GEI y cuáles a los ciclos climáticos naturales. No obstante, su impacto sobre los sectores más vulnerables de la sociedad, se hace cada vez más evidente.

Algunas de las tendencias de calentamiento, identificadas por la Organización Meteorológica Mundial (OMM, 2003), no están ligadas directamente con el cambio climático pero sí con cambios globales, que contribuyen al calentamiento global como resultado del uso de materiales modernos de construcción (que cambian las propiedades térmicas de la superficie de la tierra), del incremento de la urbanización, de la reducción de la superficie de evaporacióntranspiración (que incluso incrementa el escurrimiento), de alteraciones en el uso del suelo, de la tala de bosques y del incremento de la cantidad de brillo solar reflejado. Todas estas variaciones del sistema climático tienen notables efectos en el ciclo hidrológico, porque están estrechamente ligadas en un sistema complejo de retroalimentación, de tal forma, que los cambios en el clima inducen modificaciones en los sistemas hidrológicos, que a su vez inducen variaciones climáticas. Para facilitar el estudio de ciertos fenómenos se ha preferido simplificar esta relación a una sola vía (Kundzewicz \& Mata, 2004), sin tener en cuenta que cambios adversos en la disponibilidad, calidad y cantidad de agua, tienen notables consecuencias en el sostenimiento de la vida en el planeta y que modificaciones en variables climáticas como temperatura $y$ precipitación tienen un impacto significativo en los recursos hídricos (RH), los ecosistemas y las sociedades.

Las variaciones más importantes en el sistema climático incluyen retroalimentación en todos los sistemas hidrológicos: cambios en el vapor de agua (el efecto invernadero), en las nubes (albedo y efecto invernadero), en la temperatura del océano, almacenamiento de calor y transporte, en la escorrentía, humedad del suelo (balance de calor superficial), interacciones entre el ciclo del carbono y el ciclo del agua.

Estos cambios, sumados a la emisión de GEI pueden generar un clima más favorable en algunas regiones, mientras que en otras pueden generar devastación con tormentas severas, que conllevan pérdidas de vidas humanas y materiales; sequías, con perdida de cosechas y escasez de alimentos; variaciones en la circulación marina y la temperatura de los océanos que alteran la productividad pesquera; el deshielo de los polos, que traerá consigo un aumento en el nivel de los océanos y un incremento en la amenaza para las poblaciones costeras. En los últimos años, aspectos como la escasez de agua están cobrando una importancia significativa, así como el marcado aumento de la intensidad de la precipitación y variabilidad de eventos extremos que están teniendo impactos en las sociedades y ecosistemas que proveen bienes y servicios.

Desde hace varios años, el concepto de gestión integrada de los recursos hídricos (GIRH) viene ganando aceptación como una herramienta para asegurar un manejo equitativo, ambiental y económicamente sostenible de los recursos hídricos y de los servicios que suministran (GWP, 2002). La GIRH es una estrategia de adaptación en sistemas hídricos expuestos a fuertes variaciones espaciales y temporales a consecuencia del CC, que hacen que la oferta de agua sea cada vez más incierta mientras la demanda crece. La implementación de acciones locales depende del entendimiento y sensibilidad hacia el problema a escala global, el cual es el principal objetivo de este documento de revisión. 


\section{Impactos del cambio climático en los recursos hídricos}

El abastecimiento de agua no sólo es necesario para el sostenimiento de la vida en la Tierra, sino que sirve de insumo para diferentes actividades humanas; su conservación y óptima utilización es de gran importancia en términos de desarrollo económico. Al ser el agua el componente central del sistema climático, así como del ciclo hidrológico, cualquier cambio en el proceso de evaporación y precipitación, puede tener implicaciones importantes en el abastecimiento de agua para consumo humano, agrícola, la biodiversidad y el aumento o disminución de los niveles de los océanos (National Assesment Synthesis Team, 2002).

Según el IPCC (2001a), es muy probable que en muchas partes del mundo se presenten cambios en la cantidad, frecuencia y distribución de la lluvia, la nieve y el escurrimiento, entre el 90 y el $99 \%$ de confianza en las predicciones. Esto conducirá a cambios en la disponibilidad hídrica que implicarán una mayor competencia por los $\mathrm{RH}$, mayor intensidad y duración de las sequías, así como una mayor probabilidad de inundaciones con sus efectos en la calidad del agua. En América Latina y el Caribe se están presentando cambios importantes a causa del CC: patrones cambiantes de precipitación, como los que reportan Fernández \& Fernández (2002) en Argentina, deshielo de glaciares y una mayor frecuencia e intensidad de huracanes en el Caribe. Estos fenómenos afectan las actividades socioeconómicas que dependen fuertemente del clima o de la disponibilidad hídrica (Carvajal et al., 2005).

Según el IDEAM (2005), en Colombia la precipitación media anual es de $3000 \mathrm{~mm}$ gracias a su ubicación geográfica y a las características del relieve, lo que representa una gran riqueza de agua dulce cuando se compara con los $900 \mathrm{~mm}$ de precipitación promedio anual mundial y con los $1600 \mathrm{~mm}$ de promedio anual en Suramérica. Sin embargo, la oferta per cápita accesible anual, bajo condiciones naturales, es solo de 12.000 $\mathrm{m}^{3} / \mathrm{hab} /$ año debido a la variación temporal y espacial de los RH y a la distribución de la población en las 5 regiones del territorio nacional (MINAMBIENTE, 1996).

\subsection{Impactos sobre la cantidad}

En términos de cantidad, el CC está aumentando la competencia por agua dulce en regiones donde la disponibilidad es reducida por el incremento de la evapotranspiración como consecuencia del aumento de la temperatura del aire y por aumento de la variabilidad en la precipitación. Sin embargo, en otras áreas, los incrementos de la precipitación podrían aumentar la disponibilidad. Estos factores, la temperatura promedio, la precipitación y la humedad del suelo, son sensibles al CC mostrando también efectos sobre la demanda en la mayoría de los sectores, especialmente en la agricultura, la silvicultura y el abastecimiento de poblaciones.

El National Assesment Synthesis Team (2002), señala que el CC está presentando un impacto en todos los procesos y regímenes hidrológicos, que probablemente será mayor en el futuro. Ante ésto, cobra importancia la valoración del estado del arte de nuestro entendimiento de las relaciones entre los sistemas hidrológicos y climáticos. Es lógico entonces empezar por comprender la naturaleza de los cambios de la cantidad, intensidad, frecuencia y duración de la precipitación, en cuanto a sus estadísticos, así como la distribución temporal y espacial de las fases de precipitación (lluvia vs nieve), y la estrecha relación que estos cambios tienen con los ecosistemas y poblaciones. La comprensión de estos aspectos requiere ser abordada desde una perspectiva interdisciplinaria e integral.

Kundzewicz \& Mata (2004), reportan que en muchas regiones hay tendencias aparentes, como aumentos y disminuciones en los caudales de los ríos que pueden ser resultado del CC o de otros factores, como la variabilidad hidrológica a lo largo del tiempo. Estas precisiones son difíciles de establecer, debido al corto período de los registros disponibles. A nivel general, los cambios y la variabilidad en los caudales están en consonancia con los escenarios del CC proyectados por el IPCC (2001b), aunque la confianza al nivel de una cuenca dada es baja.

El efecto del CC en los caudales y la recarga de aguas subterráneas varía según las regiones y los escenarios, y en gran parte se ajusta a los cambios 
proyectados en la precipitación por el IPCC (2001a), que sostiene que la magnitud y la frecuencia de las inundaciones podrían aumentar en muchas regiones como consecuencia de una creciente ocurrencia de lluvias intensas que aumentarían la escorrentía, así como la recarga de aguas subterráneas. Los cambios en los caudales como consecuencia del deshielo de los glaciares han sido proyectados sobre la tasa de aumento de la temperatura, y traerían consigo la disminución del caudal de los ríos durante el verano y primavera, con la alteración de los medios de subsistencia aguas abajo, lo cual afectará la generación hidroeléctrica e intensificará los conflictos por el uso del agua, especialmente durante períodos secos y de gran demanda de agua.

\subsection{Impactos sobre la calidad del agua}

La calidad del agua es afectada por el CC de muchas formas: un aumento en la frecuencia de precipitaciones intensas ocasiona un flujo mayor de contaminantes y sedimentos en lagos y ríos, degradando la calidad del agua. De otro lado, caudales altos contribuyen a una mayor dilución de los contaminantes (Kundzewicz \& Mata, 2004), mejorando la calidad de la misma. En regiones con problemas de salinidad, la reducción en los caudales representa un problema potencial.

El aumento de la temperatura puede degradar la calidad del agua al alterar la velocidad de los procesos biogeoquímicos (algunos de degradación y autodepuración) y, en los casos más graves, puede reducir la concentración de oxígeno disuelto en la misma, afectando de esta forma las especies animales y vegetales que allí viven. En cuanto a las aguas subterráneas, la calidad puede disminuir por diversos factores que incluyen la contaminación química y microbiológica por insumos de uso agrícola, vertimientos industriales y de asentamientos humanos; así mismo, la intrusión de aguas salinas, por efecto del aumento en el nivel de los océanos, puede afectar particularmente zonas costeras. Los cambios en la calidad del agua pueden tener un enorme impacto en la salud humana, el cual depende del tamaño, densidad, ubicación y riqueza de las poblaciones. Después de ocurrida una inundación, se pueden presentar sobrecargas de los sistemas de drenaje urbano y aguas residuales, que incrementen el riesgo de ocurrencia de enfermedades hídricas (National Assesment Synthesis Team, 2002).

El fenómeno El Niño / Oscilación Sur (ENOS) produjo un incremento y expansión de enfermedades infecciosas tropicales durante el periodo 97-98 (Amarakoon et al., 2003; Poveda et al., 2001; Becker, 1997) y aumentó la incidencia de enfermedades transmitidas por el agua en gran parte de Suramérica, planteando un gran reto epidemiológico por su extensión, frecuencia, intensidad y duración (que fluctúa entre 8 meses y 5 años), así como por la dificultad de cuantificar su relación con la salud (OPS/OMS, 1998; WHO, 1996).

Los episodios extremos intensificados por el CC incrementan las tasas de mortalidad y morbilidad, como ocurrió con el huracán Mitch en Centroamérica en 1998. El incremento de la temperatura produce un aumento de las olas de calor en centros urbanos contaminados, especialmente aquellos donde las condiciones meteorológicas favorecen la estacionalidad de la misma (México DF y Santiago de Chile), trayendo como consecuencia un mayor riesgo de muertes en adultos mayores por enfermedades cardiorespiratorias (Carvajal et al., 2005).

\subsection{Aumento de los eventos extremos y desastres naturales}

El término desastre natural tiene amplia difusión, y es de uso práctico. Sin embargo, otros autores han acuñado el término desastres sociales asociado a procesos naturales (Wolansky et al., 2003). Las catástrofes naturales más importantes a lo largo de la década pasada se han triplicado, mientras que la pérdidas económicas asociadas se han incrementado 9 veces durante el mismo período (EEI, 2000). Esto se explica, en gran parte, por el incremento de la producción social de riesgo (Larrère, 1998), como consecuencia de la forma como se usa el territorio, el incremento de la densidad de población en áreas de alta vulnerabilidad, el desarrollo de actividades económicas, en sectores sujetos a riesgos, y de la reducida capacidad de enfrentar las emergencias y 


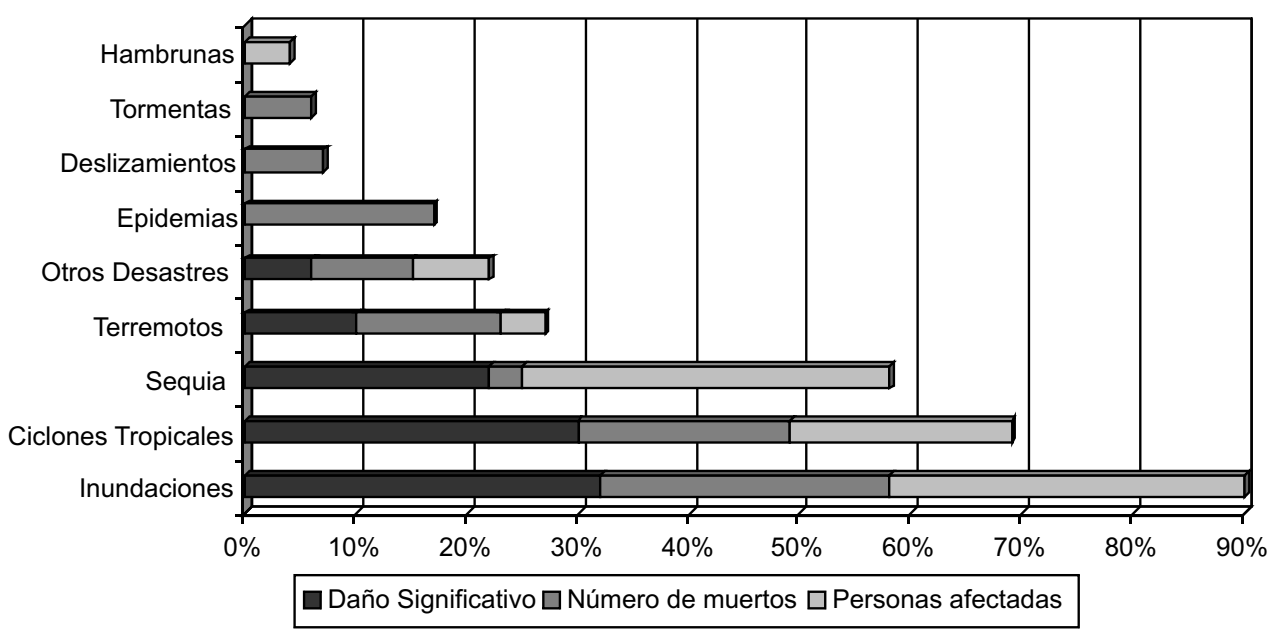

Figura 1. Efectos de los mayores desastres en el mundo entre 1963 y 1992 en términos de daño significativo, número de muertos y personas afectadas (Adaptada de Obasi, 2000).

reparar los daños consecuentes. El WDR (2003) reporta que el número de personas afectadas por desastres naturales pasó de 740 a 2000 millones entre 1963 y 1992; mientras que las pérdidas se han quintuplicado de US\$ 131 mil millones a 629 mil millones. La Figura 1 muestra los efectos de los mayores desastres en el mundo entre 1963 y 1992 en términos de daño significativo, número de muertos y personas afectadas.

Entre los años 1997 y 1998, las pérdidas relacionadas con la ocurrencia del fenómeno El Niño, fueron estimadas en US\$ 33900 millones en todo el mundo, de los cuales US\$ 18000 millones correspondieron a Latinoamérica, con mas de 117 millones de personas afectadas y 4.8 millones que perdieron sus viviendas (OPS, 2000). Según la CAF (2000), este evento produjo pérdidas tangibles estimadas en US\$ 7500 millones en la región andina, es decir, el 95\% del PIB de Bolivia en 1997, o el 32\% de las exportaciones de Venezuela; limitando notablemente la capacidad de inversión de la región. El huracán Katrina en el 2005, ocasionó uno de los peores desastres sociales asociados a fenómenos naturales en la historia de los Estados Unidos, al destruir más del $80 \%$ de Nueva Orleáns, y ocasionar graves problemas de salud pública. Más de mil personas murieron y casi medio millón fueron afectadas, y miles de ellas, desplazadas. Los daños a las viviendas y la infraestructura se han estimado en más de US\$25000 millones ( OPS, 2005).

Los desastres naturales del 2005 fueron los más catastróficos y ocasionaron las mayores pérdidas en la historia reciente. Según la OPS (2005), ese año hubo 350000 muertos y pérdidas económicas por US\$ 200000 millones. La temporada de huracanes de 2005 ha sido considerada como la más intensa de la historia con 27 tormentas tropicales y 14 ciclones. Aunque no hay consenso en torno a los factores responsables de esta altísima actividad ciclónica, hay quienes indican que se trata de un ciclo de huracanes fuertes; mientras que otros por el contrario lo adjudican al $\mathrm{CC}$, dado el incremento de la temperatura del agua en la superficie de los océanos.

En los últimos años, se presentan huracanes más intensos y frecuentes, que afectan sensiblemente las actividades socioeconómicas de la región Caribe. Webster et al. (2005), examinaron el número de ciclones tropicales y días de ciclones, así como las intensidades durante los últimos 35 años, en un ambiente creciente de temperatura superficial del océano, observando un aumento en la proporción de huracanes de categorías 4 y 5 . El aumento más grande ocurrió en el Pacífico Norte, océano Indico y el Pacífico Sudoeste, y el porcentaje de aumento más pequeño ocurrió en el 
océano Atlántico Norte. De otro lado, durante la última década, el número de ciclones y días del ciclón ha disminuido en todas las cuencas excepto el Atlántico Norte. A pesar de la falta de consenso sobre el efecto del CC en la ocurrencia de ciclones, existe un acuerdo general, en que el ciclo hidrológico es cada vez más dinámico y tendrá implicaciones en los ecosistemas y la población, generando desequilibrios económicos (CEA, 1997).

El número de refugiados ambientales está entre los 20 y 25 millones, y supera ampliamente el de personas desarraigadas por las guerras, persecuciones políticas o desastres económicos según un informe de Brauch (2005). Esta cifra podría duplicarse en los próximos 5 años. Rajendra (2005), reporta que en el 2050 el CC podría generar unos 150 millones de refugiados del clima. En India habría unos 30 millones de personas desplazadas a consecuencia del $\mathrm{CC}$ y un $17 \%$ de Bangladesh estaría inundado por el aumento del nivel de los océanos. El impacto económico inmediato es evidente, si se consideran los efectos sobre la infraestructura vial e hidráulica. Otro aspecto importante que relaciona CC y pobreza es el incremento de la tasa de migraciones y el descenso del turismo (Carvajal et al., 1998).

\section{Estrategias de adaptación: la gestión integrada de recursos hídricos}

Se prevé que con el CC, aumente la frecuencia e intensidad de los eventos catastróficos relacionados con el agua en los próximos años, siendo la población más pobre la más afectada.

El CC agravará los problemas de sequía o el acceso al agua potable, que sufren varias regiones del planeta. Actualmente, 1400 millones de personas viven en estas regiones, con menos de $1000 \mathrm{~m}^{3} /$ persona/año, y de aquí al año 2050 , entre 700 y 2800 millones de personas (según el crecimiento de la población y la gravedad del calentamiento) se verían afectadas por este fenómeno, ocasionando desplazamientos de la población, situación a la que se añadirán problemas sanitarios. En los últimos 30 años el CC ha podido causar la muerte de 150000 personas, debido al recrudecimiento de las enfermedades infecciosas y de la malnutrición. De aquí al 2020, este balance se doblará, especialmente por diarreas y malnutrición ligada a las inundaciones.

De acuerdo con el IPCC (2001c), la vulnerabilidad es mayor en sistemas hídricos mal administrados, que están bajo tensión o cuya ordenación es deficiente e insostenible, debido a políticas que desalientan el uso eficaz del recurso, la protección de la calidad del agua, o la inadecuada ordenación de las cuencas hidrográficas. La gestión tradicional de los RH, con un enfoque sectorial, no responde a las necesidades de la sociedad, agudizando la crisis del agua y aumentando el riesgo social asociado con la distribución espacio-temporal de la misma, lo cual evidencia y hace urgente la necesidad de construir una nueva visión de gestión multipropósito sustentada en acciones conducentes a la planificación, a la gestión integrada de cuencas, a los usos múltiples, y al uso armónico y equilibrado de los recursos transfronterizos e ínter-jurisdiccionales, en las cuales se sustenta la GIRH de acuerdo con GWP (2002).

La GIRH, entendida como un manejo equitativo, ambiental y económicamente sostenible de los recursos hídricos y de los servicios que proveen GWP (2002), es posiblemente la estrategia fundamental de adaptación al CC, al permitir resiliencia ante los efectos hidrológicos de los eventos extremos y a las nuevas incertidumbres, disminuyendo la vulnerabilidad. La habilidad de un sistema de ajustarse al cambio climático (incluida la variabilidad del clima y sus extremos) para moderar posibles daños, aprovecharse de oportunidades o enfrentarse a las consecuencias, se entiende como capacidad de adaptación (IPCC, 2001b).

Por otra parte, la GIRH es un proceso de desarrollo coordinado de los recursos naturales, que maximiza el bienestar social y económico de manera equitativa y sin comprometer la sostenibilidad de los ecosistemas vitales. Esto supone superar los viejos paradigmas donde los enfoques referentes al suministro de agua tienen mayor difusión que aquellos referidos a la demanda (que alteran la exposición a la tensión), 
aunque estos últimos han venido ganando una mayor atención. Sin embargo, la capacidad de aplicar respuestas de ordenación eficaces está desequilibradamente distribuida por todo el mundo y es baja en muchos países con economías en transición y en desarrollo.

Las estrategias de adaptación a los efectos del CC en términos de una GIRH son diversas y pueden ser aplicadas a todos los niveles: van desde cambios en la operación de diques y reservorios, la reevaluación de los supuestos básicos de la ingeniería usados para el diseño y la construcción de infraestructura, la conservación del recurso agua, el reuso de agua residual tratada, el incremento de los precios, que se espera se traduzcan en aumentos en la eficiencia del uso, entre otros. Kundzewicz \& Mata (2004) recogen algunas de las estrategias de adaptación propuestas mundialmente:

- Incremento de la habilidad de intercambio entre los sectores.

- Uso de mecanismos de mercadeo para aumentar eficacia del uso de agua.

- Incorporación de cambios potenciales en la demanda y la oferta, en la planificación y en el diseño de infraestructura.

- Reubicación de comunidades y estructuras fuera de las planicies inundables.

- Identificación de formas sostenibles de manejar la oferta disponible, incluyendo agua subterránea, superficial y los efluentes.

- Restauración y mantenimiento de las fuentes de agua como una estrategia integrada para el manejo de la calidad y la cantidad del agua.

- Reducir el arrastre de sedimentos y carga de nutrientes, limitar las inundaciones y reducción de la temperatura del agua en fuentes que han sido afectadas por la urbanización y la silvicultura.

- Reuso de aguas residuales municipales (domiciliarias e industriales).
- Mejoramiento del manejo del drenaje urbano y promoción de sistemas para recolección de agua lluvia que refuercen los suministros de agua urbanos.

- Incremento del uso de herramientas para la predicción del clima y posibles efectos de fenómenos como El Niño, que pueden ayudar a reducir daños y perjuicios asociados con los eventos extremos.

- Mejoramiento del monitoreo de datos para la modelación climática e hidrológica con el fin de ayudar al entendimiento de los impactos relacionados con el agua y las posibles estrategias de manejo.

Debido a que muchos de los impactos del CC no son predecibles, se requieren además arreglos institucionales más flexibles a fin de que se puedan adaptar a las cambiantes condiciones que no sólo incluyen el clima, sino también otras generadas por el cambio global. Una consideración importante en cuanto a las estrategias de adaptación es que los humanos tenemos un gran potencial de adaptación al cambio, a diferencia de los ecosistemas que son más vulnerables.

\section{Conclusiones y recomendaciones}

El CC está generando un impacto importante en los ecosistemas vitales y las actividades socioeconómicas, que implica la necesidad de evaluar e implementar políticas de adaptación para disminuir la vulnerabilidad de la sociedad. El CC produce un efecto desestabilizador en el ciclo hidrológico que, sumado al manejo insostenible de los recursos naturales, intensificará la ocurrencia de desastres naturales. Proponer soluciones es uno de los mayores retos de la GIRH en los próximos años (II Foro Mundial del Agua, 2000). El sector hídrico ha prestado poca atención al $\mathrm{CC}$, por enfocarse en aspectos más urgentes, como el saneamiento básico, el agua potable y la reducción de la contaminación. No obstante, es necesario considerar su impacto a largo plazo. La experiencia indica que será mucho más caro y difícil enfrentar sus impactos una vez producidos, que prepararse para estar adaptados a ellos. 
La reducción de la disponibilidad de RH afectará el desarrollo de actividades que, como la agricultura, tienen alta demanda de agua, lo cual implica el desplazamiento de zonas agrícolas. Este hecho obliga al desarrollo y adaptación de especies a las nuevas condiciones y a la previsión de nuevas amenazas alimentarias. Los aumentos de la temperatura y la reducción de los RH en los valles interandinos de Colombia, pueden generar el desplazamiento de la frontera agrícola hacia zonas más altas, caracterizadas por temperaturas más moderadas, que constituyen fuentes de agua y zonas de reserva natural.

\subsection{Implicaciones del cambio climático en el manejo de los recursos hídricos}

Es necesario extender la transferencia de funciones entre los sistemas climáticos $\mathrm{e}$ hidrológicos a fin de abarcar los RH y su gestión. Esto puede interpretarse como una cascada: del clima a la hidrología, de la hidrología a la gestión de RH. Deben evaluarse impactos regionales y sectoriales relacionados con el agua a causa del CC (National Assesment Synthesis Team, 2002). Los impactos del cambio climático no son sólo visibles en la disponibilidad de recurso de agua, sino también en la demanda de agua y los usos. La distribución de la población, la agricultura de riego, el uso de agua a nivel urbano e industrial son los principales problemas relacionados con la disponibilidad de agua.

Las consecuencias de cambios en la intensidad y frecuencia y en particular los eventos hidrometeorológicos extremos, como las inundaciones y las sequías, podrían presentarse en muchos sectores y virtualmente, en todas las regiones. Hasta ahora el debate se ha enfocado en las cuestiones propias de los recursos hídricos tales como el suministro de agua, el manejo de inundaciones y sequías, la irrigación. Pero no sólo se debe enfatizar en los aspectos físicos de la hidrología sino que deben tenerse en cuenta también los factores que afectan la vulnerabilidad social y económica. Estos incluyen los aumentos en la población y en la demanda. Hay una necesidad urgente de mejorar la eficiencia en el uso del agua para agricultura.
WMO-UNEP (1997) presenta varias directrices para reducir la vulnerabilidad potencial de los sistemas hídricos al CC: la determinación de tarifas, iniciativas orientadas a la eficiencia hídrica, mejoras ingenieriles y estructurales en la infraestructura del suministro de agua, políticas agrícolas y planificación/gestión urbanística. A nivel nacional/regional, sería prioritario dar más preponderancia a la gestión integrada e intersectorial de los $\mathrm{RH}$, utilizar las cuencas hidrográficas como unidades de gestión de recursos, o fomentar unas prácticas de determinación de precios y de gestión apropiadas. El aumento de la demanda, la prevalencia y la sensibilidad de muchos sistemas de gestión de agua simples frente a las fluctuaciones de precipitación y escorrentía, y las considerables cantidades de tiempo y dinero que se requieren para poner en marcha muchas de las medidas de adaptación, hacen que el sector de RH sea vulnerable al CC en varias regiones y países.

\subsection{Implicaciones políticas del cambio climático}

Los países en desarrollo son más vulnerables debido a la escasez de recursos técnicos, financieros y de gestión para adaptarse a las situaciones de escasez o implementar medidas de adaptación. Los impactos dependerán del estado comparativo del sistema de abastecimiento de agua y de la capacidad de la GIRH para responder al crecimiento de la población, así como a los cambios en la demanda de tecnologías y de las condiciones económicas, sociales y legislativas. Cada vez se impone el enfoque de la GIRH, para responder a estas realidades cambiantes (Carvajal, 2005) y la adaptación al CC ofrece una oportunidad para innovar en la gestión hídrica.

Los cambios en la precipitación pueden afectar una variedad de aspectos relacionados con la planificación y el diseño de estructuras hidráulicas, la gestión de cuencas, el control de sequías e inundaciones, la planificación urbana y el desarrollo industrial.

Existen además otras implicaciones más allá de los problemas de suministro de agua. La política agraria requerirá inversiones tendientes a anticipar la selección de las épocas de cosecha en 
función de las variaciones espacio-temporales del RH. La política forestal necesitará tener en cuenta la mitigación de la erosión en áreas donde la precipitación se predice que es alta y el tratamiento de aguas residuales necesitará tratarse en contextos donde la precipitación es intensa. Se han reconocido además futuras necesidades de investigación:

1. Desarrollo de modelos espaciales para permitir mayor precisión en las predicciones de cambio de clima.

2. Investigación agrícola en cultivos resistentes a la sequía y a la inundación.

3. Investigación en ciencias sociales sobre los cambios de calidad y cantidad de agua al nivel de las cuencas, causados por aumentos en la precipitación.

4. Análisis de datos de descargas contaminantes en los ríos.

5. Disponibilidad de agua, incluyendo reuso de aguas residuales.

\section{Referencias bibliográficas}

Amarakoon, A. M. D., Chen, A.A., Rawlins, S.C., \&Taylor,M.A.(2003). Dengue epidemics-its association with precipitation and temperature, and its seasonality in some Caribbean countries. The University of the West Indies, Jamaica.

h t t p : / / chiex.net/documents / CHR S 2004_submitted.doc

Becker, D. (1997). Global Warming: Central debate number three.

Brauch, H.G. (2005). Threats, challenges, vulnerabilites and risks in environmental and human security. Studies of the University: Research, Counsel, Education (SOURCE) No.1/ 2005, UNU-EHS (United Nations University, Institute for Environment and Human Security).
CAF (Corporación Andina de Fomento). (2000). Las lecciones de El Niño: memorias del fenómeno El Niño 1997-1998: retos y propuestas para la región andina: Ecuador. CAF, Documento 15907, Caracas, Venezuela.

http://www.crid.or.cr/digitalizacion/pdf/spa/ doc15907/doc15907.htm

Carvajal, Y., Jiménez, H., \& Materón, H. (1998). Incidencia del fenómeno del Niño en la hidroclimatología del valle del río Cauca, Colombia. En: E. Cadier, G. Gómez, R. Galarraga, C. Fernández-Jáuregui (editores), Consecuencias climáticas e hidrológicas del evento El Niño a escala regional y local: Incidencia en América del Sur (UNESCO-PHI).

http://www.unesco.org.uy/phi/libros/enso/ carvajal.html

Carvajal, Y. (2005). Desafios de la formación de postrado en ingeniería del agua para América Latina. En Memorias de la Conferencia Internacional Agua 2005 (De la acción local a las metas globales), Universidad del Valle, Cali, Colombia.

Carvajal, Y., Restrepo-Tarquino, I., \& Tucci, C. (2005). El Cambio climático y las estrategias de desarrollo para América Latina. En Memorias de la Conferencia Internacional Agua 2005 (De la acción local a las metas globales), Universidad del Valle, Cali, Colombia.

CEA (Canadian Environmental Agency). (1997). Environmental Issues.

CEPAL (2000). Panorama del impacto ambiental de los recientes desastres naturales en América Latina y el Caribe. Documento preparado para el Comité Técnico Interagencial del Foro de Ministros de Medio Ambiente de América Latina y el Caribe, PNUMA, México, D.F., México.

EEI (Emerging Environmental Issues). (2000). Sacando a relucir temas relacionados con el medio ambiente. Documento presentado a la Sesión Especial de Ministros de PNUD, Malmo, Suecia. 
Fernández, H.W., \& Fernández, B.L. (2002). Influencia de ENOS en el régimen de las precipitaciones en el cono sur de América. Ingeniería Hidráulica en México, 17 (3), 5-16.

GIECC (Grupo Intergubernamental de Expertos sobre el Cambio Climático). (1997). Impactos regionales del cambio climático: evaluación de la vulnerabilidad. Resumen para responsables de políticas, Ginebra, Suiza.

GWP (Global Water Partnership). (2002). Agua para el siglo XXI: de la visión a la acción. Global Water Partnership, Estocolmo, Suecia; Buenos Aires, Argentina.

IDEAM (Instituto Colombiano de Hidrología, Meteorología y Estudios Ambientales). (2005). Estudio de aguas de Colombia. Versión actualizada. Santa Fe de Bogotá, Colombia.

IPCC (Intergovernmental Panel on Climate Change). (2001a). Climate change 2001: the scientific basis. Contribution of Working Group I to the third assessment report of the intergovernmental panel on climate change. J.T. Houghton, Y. Ding, D.J. Griggs, M. Noguer, P.J. Van der Linden, X. Dai, K. Maskell, \& C.A. Johnson (editors). Cambridge University Press, United Kingdom.

IPCC (Intergovernmental Panel on Climate Change). (2001b). Climate change 2001: impacts, adaptation, and vulnerability. contribution of Working Group II to the third assessment report of the intergovernmental panel on climate change. J.J. McCarthy, O.F. Canziani, N.A. Leary, D.J. Dokken, \& K.S. White (editors). Cambridge University Press, United Kingdom.

IPCC (Intergovernmental Panel on Climate Change). (2001c). Climate change 2001: mitigation. contribution of Working Group III to the third assessment report of the intergovernmental panel on climate change. B. Metz, O. Davidson, R. Swart, \& J. Pan. Cambridge University Press, United Kingdom.
Kundzewicz, Z. W., \& Mata, L.J. (2004). Concept paper on cross-cutting theme: water. Progress of Working Group II towards the IPCC Fourth Assessment Report (Ar4).

http://www.ipcc.ch/activity/cct6.pdf

Larrère, R. (1998). L'erosion, l' avalanche et le forestier. En: Le sistemazioni idraulico-forestali: tra revisione e sviluppo (Quaderni di idronomia montana 17). Editoriale Bios, Cosenza, Italia, p. 13-30.

MINAMBIENTE (Ministerio del Medio Ambiente). (1996). Lineamientos de política para el manejo integral del agua. Santa Fe de Bogotá, Colombia.

h t t p : / / w w w. id e a m.gov.co/a p c aa/img_upload/467567db4678d7b443628f8bc21 $5 f 32 \mathrm{~d} /$ Lineamientos_Agua.pdf

National Assesment Synthesis Team. (2002). Water sector from the overview of climate change impacts on the US: the potential consequences of climate variability and change. US Global Change Research Program.

http://www.usgcrp.gov/usgcrp/Library/nationala ssessment/16WA.pdf

Obasi G.O.P. (2000). Predecir los desastres naturales para mitigar sus efectos. Memorias de la Conferencia Cómo enfrentar los desastres naturales: una cuestión de desarrollo. Organización Meteorológica Mundial (OMM), Banco Interamericano de Desarrollo, New Orleáns, Estados Unidos.

http://www.crid.or.cr/digitalizacion/pdf/spa/ doc14165/doc14165.htm

OMM (Organización Meteorológica Mundial). (2003). Declaración de la OMM sobre la situación del clima mundial en 2003. 2003: El tercer año más caliente, Ginebra, Suiza.

http://www.meteored.com/ram/numero17/ omm.asp

OPS (Organización Panamericana de la Salud) / OMS (Organización Mundial de la Salud). (1998). Repercusiones sanitarias del Fenómeno del Niño. Washington, D.C., Estados Unidos. 
OPS (Organización Panamericana de la Salud). (2000). Crónicas de desastres - Fenómeno El Niño 1997-1998. Washington, Estados Unidos.

http://cidbimena.desastres.hn/docum/ops/ publicaciones/who 70s/who70s.3.htm

OPS (Organización Panamericana de la Salud). (2005). Desastres, preparativos y mitigación en las Américas. B ole tín 101 . http://www.paho.org/spanish/DD/PED/ ped1005s.pdf

Poveda, G., Rojas W., Quiñones, M. L., Velez, I. D., Mantilla, R. I., Ruiz, D., Zuluaga, J. S., \& Rua, G. L. (2001). Coupling between annual and ENSO timescales in the Malaria-climate association in Colombia. Environmental Health Perspectives, $109(5), 489-493$.

Rajendra, P. (2005). Avoiding dangerous climatic change. Exeter, Inglaterra.

WDR (World Disasters Report), (2003). World Disasters Report Focus on Ethics and Aid. J. Walter (editor). International Federation of Red Cross and Red Crescent Societies, Kumarian Press.

Webster, P. J., Holland, G. J., Curry, J. A., \& Chang, H. R. (2005). Changes in tropical cyclone number, duration and intensity in a warming environment. Science Magazine 309 (5742), 1844-1846.

http://www.sciencemag.org/cgi/content/full/309/ $5742 / 1844$

WHO (World Health Organization). (1996). Climate change and human health 1996. Document who/ehg/96.(7).

WMO (World Meterological Organization)UNEP (United Nations Environment Programme). (1997). Impactos regionales del cambio climático: evaluación de la vulnerabilidad. Resumen para responsables de políticas. Grupo Intergubernamental de expertos sobre el cambio climático. Ginebra, Suiza.
Wolansky, S., Corzo, H., Valsagna, A., \& Morbidoni, N. (2003). Las inundaciones en Santa $\mathrm{Fe}$. Desastres naturales y mitigación del riesgo. Volumen 2. Centro de Publicaciones, Secretaria de Extensión, Universidad Nacional del Litoral, Santa Fe, Argentina. 